\title{
Editorial
}

\section{The Inflammatory Effector Cell Pattern in Asthma and Chronic Obstructive Pulmonary Disease - What Is It Good for?}

\author{
Claus Kroegel ${ }^{\mathrm{a}}$ Petros Bakakos $^{\mathrm{b}}$ \\ a Department of Pneumology and Allergy, Internal Medical Clinic I, Friedrich Schiller University, Jena, Germany; \\ b 1st University Department of Respiratory Medicine, Athens, Greece
}

The two obstructive airway diseases bronchial asthma and chronic obstructive pulmonary disease (COPD) represent major global causes of disability and death. Asthma is commonly considered an allergic disease that develops in the first decade of life and is characterized by the presence of reversible airflow obstruction. In contrast, COPD develops later in life, is related to tobacco smoking and characterized by the presence of incompletely reversible or irreversible airflow obstruction as well as by accelerated lung function decline. However, it has long been recognized that asthma and COPD are heterogeneous and may show a considerable overlap in respect to clinical presentation, lung function, response to treatment and certain features of the underlying inflammation [1].

In respect to the associated inflammation, two major patterns have been defined based on the predominance of eosinophils or neutrophils detected in sputum, bronchoalveolar space and airway tissue. While an eosinophilic pattern can usually be found in asthma and is associated with increased serum immunoglobulin E concentration [2], the neutrophilic pattern is preferentially observed in severe manifestations of the disease. In contrast, COPD is associated with a neutrophilic pattern whereas the presence of eosinophils indicates recurrent infections and accelerated lung function decline $[3,4]$. Although several hypotheses have been proposed to explain the variable presence of the two inflammatory effector cell types, the nature of the eosinophilic/neutro-

\section{KARGER}

Fax +4161306 1234

E-Mail karger@karger.ch

www.karger.com (c) 2011 S. Karger AG, Basel

0025-7931/12/0831-0017\$38.00/0

Accessible online at:

www.karger.com/res philic overlap observed between asthma and COPD has not yet been conclusively understood.

Several reasons may account for overlapping features of asthma and COPD. First, asthma and COPD are common conditions affecting between 5 and $15 \%$ of the population each. Therefore, overlap is likely to occur by chance alone. Second, the two conditions may share common origins or risk factors, such as respiratory infections or smoking, leading to epigenetic alterations with different inflammatory patterns [1]. Third, the predominance of neutrophils or eosinophils observed in either condition may represent an epiphenomenon with little pathogenic or diagnostic relevance. The predominance of a given effector cell may simply be due to comorbidity, exogenous factors or treatment, such as corticosteroids, which are known to favor an increase in neutrophils while reducing the number of eosinophils [5].

A new study by Bafadhel et al. [6] published in this issue of Respiration uses a different approach to look at the overlap of asthma and COPD in respect to inflammation. First, the report explores airway inflammation as well as cytokine and chemokine expression in stable adult asthma and COPD patients using a novel two-step sputum processing method with dithiothreitol supernatants and cytospin preparations, allowing analysis of a broad spectrum of cytokines previously not accessible. In addition, sputum neutrophils were significantly lower in mild asthma compared to moderate/severe disease whereas sputum eosino- 
Table 1. Summary of the differences in sputum concentration of cytokines/cytokine receptors, chemokines and MMPs between asthma and COPD and between the eosinophil- and neutrophil-dominated patterns

\begin{tabular}{|c|c|c|c|}
\hline & Cytokines/cytokine receptors & Chemokines & MMPs \\
\hline Increased concentrations of COPD versus asthma & \multicolumn{2}{|l|}{ IL-8, TNFRI, TNFRII } & \multirow[t]{2}{*}{ MMP8, MMP9 } \\
\hline Increased concentrations of asthma versus COPD & \multicolumn{2}{|l|}{ IL-5 } & \\
\hline versus the eosinophilic pattern & IL-6, IL-8, TNF- $\alpha$, TNFRI, TNFRII & CCL5 & \multirow[t]{2}{*}{ MMP8, MMP9 } \\
\hline Net difference & IL-5, IL-6, IL-8, TNF- $\alpha$ & CCL5 & \\
\hline
\end{tabular}

Data are from Bafadhel et al. [6].

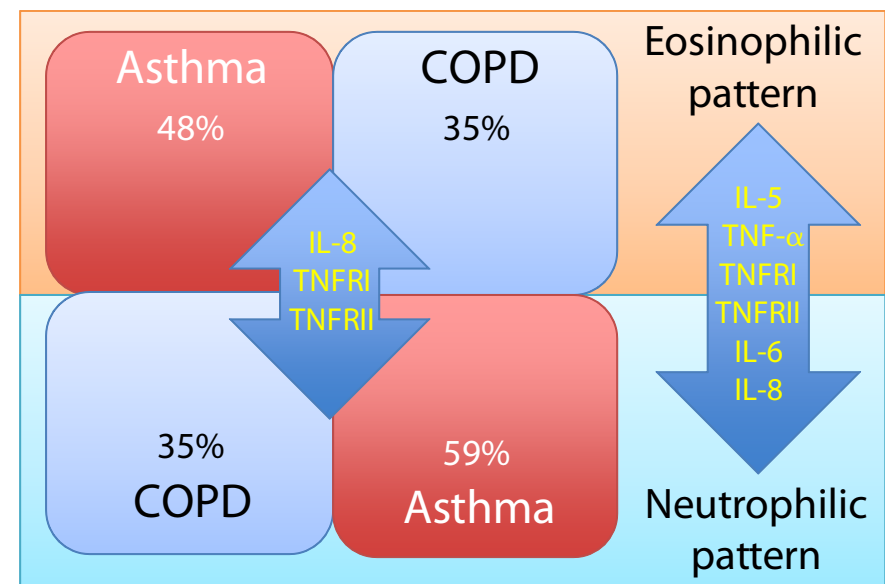

Fig. 1. Semiquantitative representation of the eosinophilic and neutrophilic pattern as well as of differences in sputum cytokine and chemokine concentration in both asthma versus COPD and eosinophilic versus neutrophilic inflammation. Percentages indicate the proportion of the inflammatory pattern observed in COPD or asthma, respectively. The two-sided arrows indicate the difference in mediator concentration. Data are from Bafadhel et al. [6].

phils were significantly lower in mild/moderate COPD compared to severe disease.

Second, and in contrast to most other studies in this field, the investigation compares potential differences between the two inflammatory patterns (eosinophilic vs. neutrophilic) independent from the underlying disease. Using this dual approach, the study shows that differential mediator expression is more pronounced between eosinophilic and non-eosinophilic subphenotypes independent of clinically defined disease than between asthma and COPD. While asthma and COPD differ in respect to interleukin (IL)-8, tumor necrosis factor receptor I (TNFRI), TNFRII, matrix metalloproteinase 8 (MMP8) and MMP9, the significant difference between the eosinophil- and 气neutrophil-dominated pattern includes IL-5, IL-6, IL-8, TNFRI, TNFRII, CCL5, MMP8 and MMP9 (table 1; fig. 1).

What does this finding of the study tell us? At first glance, the results appear to support the suggestion that both asthma and COPD may share common origins with differences in phenotypic presentation due to disease evolution or interaction between endogenous and exogenous factors. However, at second look, the data may suggest something different. The results of the investigation by Bafadhel et al. [6] demonstrate that in severe clinical expressions of either disease the respective effector cells tend to become more prominent (e.g., eosinophils in COPD and neutrophils in asthma). In more severe clinical manifestations of obstructive airway disease, comorbidity (bronchiectasis, postnasal drip, gastroesophageal reflux, immunoglobulin deficiency, allergic sensitization) and environmental exposure (tobacco smoke, various pollutants or particulates) may be present. In addition, more severe clinical manifestations are likely to require regular treatment using multiple drugs given in higher doses compared to mild expression of the diseases, including oral corticosteroids, indicating that exogenous factors such as smoking may influence the inflammatory pattern [7]. Therefore, the variable predominance of either neutrophils or eosinophils observed in asthma and COPD may primarily be a consequence of multiple factors active in more severe disease.

Considering the substantial complexity resulting from coexisting multiple conditions, one may ask whether effector cells represent an adequate parameter to define subtypes of asthma and COPD. Likewise, the significant differences in respect to several cytokines and associated receptors observed by Bafadhel et al. [7] comparing eosinophilic and neutrophilic predominance may reflect the immune response underlying neutrophil and eosinophil recruitment/involvement shaped by the multitude of coactive endogenous or exogenous factors. In other 
words, a distinction of asthma or COPD on the basis of the dominating effector cell may be, at best, applicable in mild disease without comorbidity where other factors are not active.

This view is supported by a bias inherent to studies comparing asthma with COPD. Patients with COPD are by nature of the disease older than those suffering from asthma, limiting direct comparison of the two conditions. In addition, depending on the study population, patients with overlapping asthma and COPD, smokers or those with atopy are excluded from clinical trials. For instance, a study on the efficacy of inhaled corticosteroids in asthma would exclude smokers with asthma because of the difficulty in separating asthma from COPD in smokers with obstructive lung disease. As up to one third of people with asthma are smokers, a substantial proportion of the population are excluded from randomized controlled trials. Therefore, a proportion of patients with coexisting eosinophilic-neutrophilic pattern may remain unnoticed.

Of unselected subjects with stable obstructive airway disease (asthma and/or COPD), two thirds exhibited an overlap syndrome of asthma with COPD, and only 16 and $21 \%$, respectively, could be definitively categorized as asthma and COPD based on lung function parameters [1]. The participants with overlap syndrome and COPD are predominantly ex-smokers, and the rate of allergic sensitization was $64 \%$ in overlap syndrome and $25 \%$ in COPD alone. Eosinophil numbers are significantly increased in asthma compared with healthy subjects but not different to COPD. Together, these data confirm that the overlapping presence of eosinophils and neutrophils is a common feature, frequently due to coexisting atopy.

Neutrophils and eosinophils are effector cells operating at the very end of the distal immune responses [8]. They proliferate and infiltrate tissue as a consequence of a multitude of specific or non-specific factors, including infections, drugs or toxins. Thus, the number of effector cells represents, by nature, a rather non-specific parameter which does not allow to draw conclusions regarding the underlying pathogenic mechanism. The limited applicability of effector cells in obstructive airway disease is supported by the fact that independent of an eosinophilic or neutrophilic predominance, there are still distinct differences between the diseases regarding the more proximal immunological features. For instance, asthmatic airway inflammation is characterized in most cases by an increased number of activated T lymphocytes, particularly CD4+ Th2 lymphocytes, eosinophils and mast cells. In stable COPD, airway inflammation is characterized by an increased number of CD8+ T lymphocytes, activated macrophages and neutrophils [9].

Taken together, we are still far from understanding the nature of overlap between asthma and COPD. However, the data presented by Bafadhel et al. [7] and others indicate that eosinophilic and neutrophilic inflammation may not be an ideal parameter to differentiate asthma from COPD or to define subtypes of these conditions. In fact, the findings presented a challenge to the categorization of patients into eosinophilic and neutrophilic inflammation as a means to define subtypes of either asthma or COPD. As effector cells represent the very periphery of multiple immune responses to various specific and non-specific factors (environmental exposures, coexisting diseases and treatment), they are neither sensitive nor specific. Considering the complexity of immunological and morphological alterations underlying COPD or asth$\mathrm{ma}$, reliable categorization is likely to require an integrated assessment of multiple clinical, functional, morphologic, immunological, molecular and genetic factors rather than an ubiquous effector cell.

\section{References}

1 Gibson PG, Simpson JL: The overlap syndrome of asthma and COPD: what are its features and how important is it? Thorax 2009; 64:728-735.

-2 Oryszczyn M-P, Bouzigon E, Maccario J, et al: Interrelationships of quantitative asthma-related phenotypes in the Epidemiological Study on the Genetics and Environment of Asthma, Bronchial Hyperresponsiveness, and Atopy. J Allergy Clin Immunol 2007;119:57-63.

- 3 D'Armiento JM, Scharf SM, Roth MD, et al: Eosinophil and T cell markers predict functional decline in COPD patients. Respir Res 2009;10:113-1126.
4 Chang J, Mosenifar Z: Differentiating COPD from asthma in clinical practice. J Intensive Care Med 2007;22:300-309.

5 Burrows B, Knudson R, Lebowitz M: The relationship of childhood respiratory illness to adult obstructive airway disease. Am Rev Respir Dis 1977;115:51-60.

-6 Bafadhel M, McCormick M, Saha S, et al: Profiling of sputum inflammatory mediators in asthma and chronic obstructive pulmonary disease. Respiration 2012;83:3644.
7 Bafadhel M, Saha S, Siva R, McCormick M, et al: IL-5 concentration is associated with sputum eosinophilia and attenuated by corticosteroid therapy in COPD. Respiration 2009; 78:256-262.

8 Kroegel C, Reissig A, Mock B: Eosinophilic pneumonia; in: Respiratory Medicine, ed 3. Harcourt Health Service, London, New York, 2002, pp 1643-1659.

$\checkmark 9$ Welte T, Groneberg DA: Asthma and COPD. Exp Toxicol Pathol 2006;57(suppl 2):35-40. 